\section{MS12-P2 New approach to protein crystallization. Investigation of various crystallization stages of lysozyme.}

Yuliya A. Dyakova ${ }^{1,2}$, Alexander E. Blagov ${ }^{1,2}$, Margarita A. Marchenkova $^{1,2}$, Yury V. Pisarevskiy ${ }^{1,2}$, Pavel A. Prosekov ${ }^{1,2}$, Vladimir V. Volkov ${ }^{1}$, Mikhail V. Kovalchuk ${ }^{1,2,3}$

1. Shubnikov Institute of Crystallography of Russian Academy of Sciences

2. National Research Centre "Kurchatov Institute"

3. St. Petersburg State University

email: juliaadi@yandex.ru

Nowadays protein crystallization is an extensive area of the scientific research, but a very important and difficult challenge in this area is to find the proper crystallization conditions. Currently the crystallization conditions are usually obtained by trial-and-error methods; hence, the possibility to obtain high X-ray diffraction (XRD) quality protein crystals is not so predictable.

In this work the processes of lysozyme crystals growth on different substrates have been studied in situ by XRD technique. Lysozyme crystals on substrates were grown using the sitting-drop method in a specially designed sealed crystallization cell with chemically fixed inner environment. This crystallization cell is suitable for optical and XRD studies in a wide incident-angle range, which enables researchers to perform in situ studies of the protein crystals growth and their properties in the native state (1).

An initial crystallization stage is of great importance for both the crystallization conditions search and obtaining high-quality crystals.

We proposed and experimentally demonstrated a deep relation between an oligomer formation and the best crystallization conditions for tetragonal lysozyme.

We selected elements in the crystal structure of tetragonal lysozyme crystals, which are ordered oligomers, and assumed that these oligomers can function as growth units in crystal growth.

The study of lysozyme conformation was conducted under different temperatures and concentration of protein. We also investigated the effect of substitution in the crystallization solution of ordinary water $\left(\mathrm{H}_{2} \mathrm{O}\right)$ to heavy water $\left(\mathrm{D}_{2} \mathrm{O}\right)$.

The results showed a noticeable presence of lysozyme dimers and octamers under crystallization conditions and the total absence of oligomers under conditions where crystal growth was impossible. Fig. 1 We identified the structures of possible crystal growth units of tetragonal lysozyme and revealed the relationship between the formation of certain types of oligomers in solution and the optimum crystallization conditions. Hence, our approach could be useful for studying protein crystallization mechanisms (2).

Based on the results presented in the paper, thin films were prepared by lysozyme area of about $1 \mathrm{~cm}$ square.

This study was supported in part by the RFBR (project no. 15-29-01142_ofi_m, 16-32-60144_mol_a_dk)

1- Kovalchuk, M. V. and all // Crystallogr. Rep. 2014, 59,679

2- Mikhail V. Kovalchuk and all // Crystal Growth \& Design. 2016, 16 (4), 1792

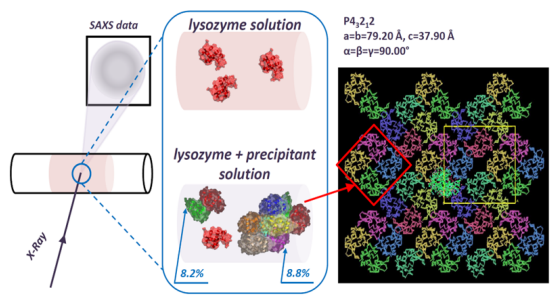

Figure 1. Scheme study of the initial stages of crystallization of lysozyme by SAXS. A projection of the tetragonal HEWL structure perpendicular to the 4 -fold axis. The octamers are proposed as growth 'building blocks'.

Keywords: crystal growth unit, protein solution, small angle X-ray scattering, molecular modelling, lysozyme, X-ray diffraction 